\title{
Nanoscale Coordination Polymer Based Nano-Vaccine for Tumor Immunotherapy
}

He Zhao ${ }^{1+}$, Jun $\mathrm{Xu}^{2+}$, Yan $\mathrm{Li}^{1}$, Xinxian Guan ${ }^{1}$, Xiao Han ${ }^{2}$, Yunyun $\mathrm{Xu}^{1}$, Huiting Zhou ${ }^{1}$, Rui Peng ${ }^{2}$, Jian Wang ${ }^{1 *}$, Zhuang Liu $^{2 *}$

1 Children's Hospital of Soochow University, Pediatric Research Institute of Soochow University, Suzhou, Jiangsu 215123, China

2 Institute of Functional Nano \& Soft Materials (FUNSOM), Jiangsu Key Laboratory for Carbon-Based Functional Materials \& Devices, Soochow University, Suzhou, Jiangsu 215123, China + These authors contributed equally to this work.

*Email: wj196312@vip.163.com, zliu@suda.edu.cn 
A

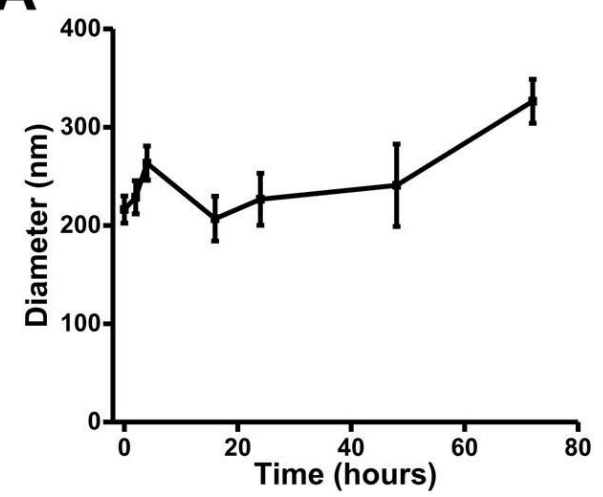

C

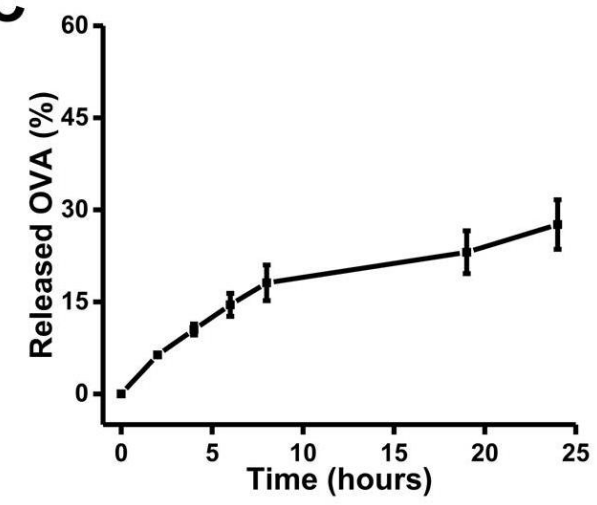

B

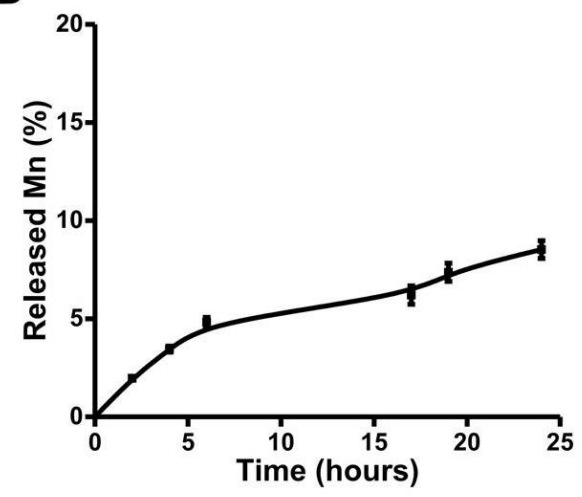

D

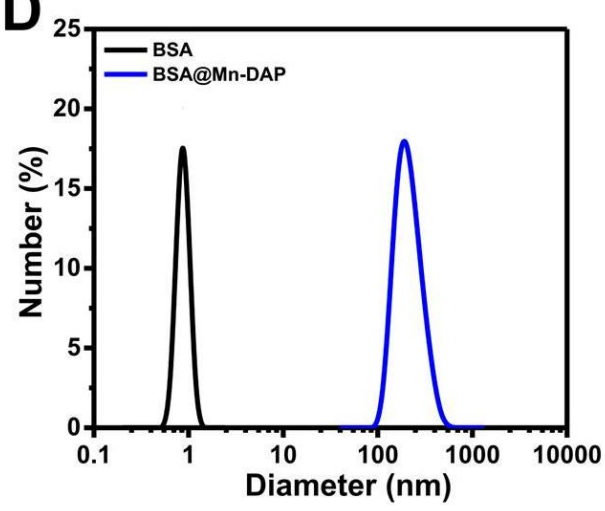

Figure S1. (A) The diameter changes of the nano-vaccine in the $\mathrm{pH} 7.4$ solution with $10 \%$ serum at different time points. (B\&C) The $\mathrm{Mn}^{2+}$ (B) or OVA (C) release of OVA@Mn-DAP in the pH 7.4 solution at various time points. (D) The size distribution of BSA and BSA@Mn-DAP nanoparticle. 
A
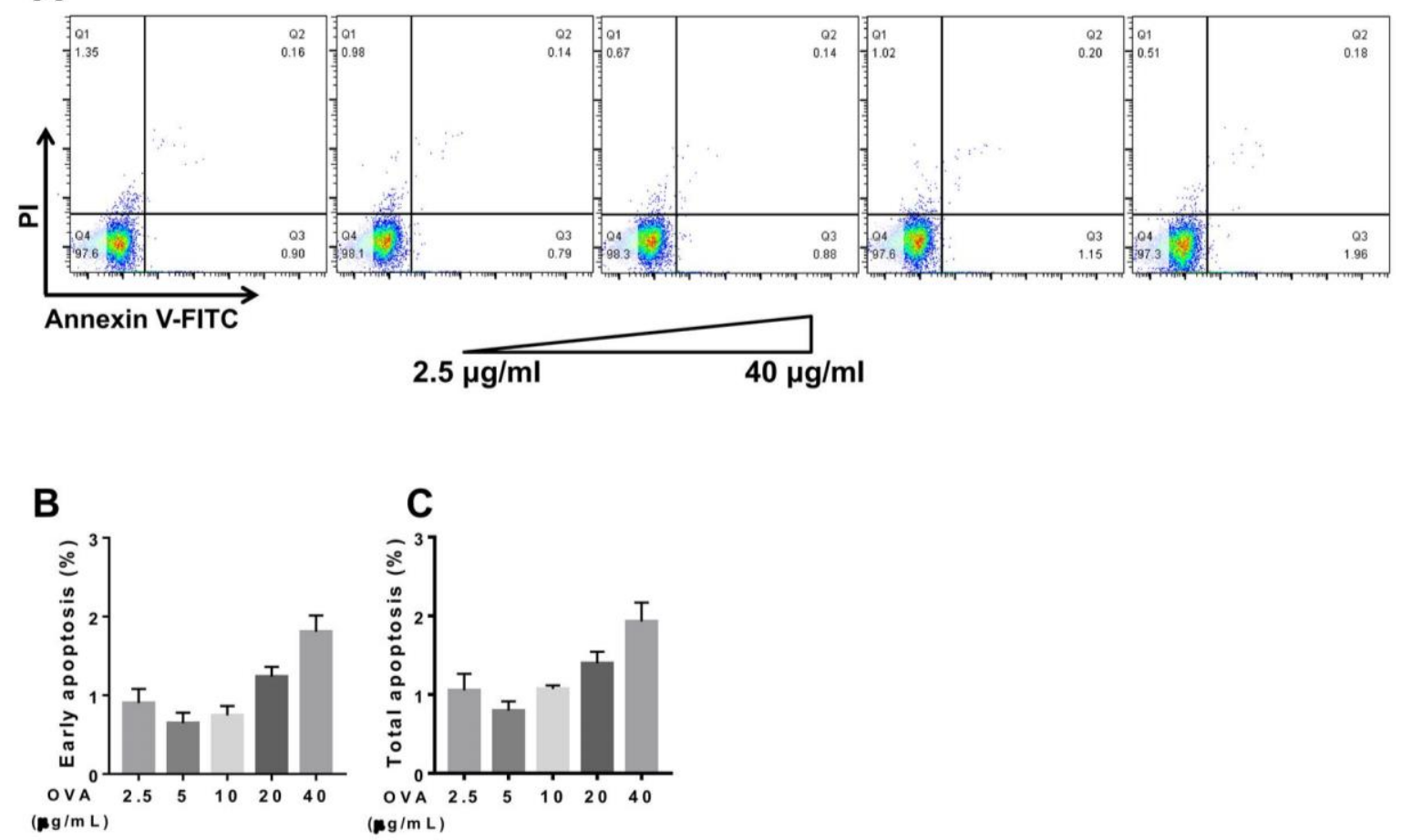

Figure S2. In vitro test to evaluate the apoptosis of DCs induced by OVA@Mn-DAP nanoparticles. Flow cytometry plots (A) and the statistical results (B\&C) of DCs incubated with various concentrations of OVA@Mn-DAP nanoparticle for 24 hours are presented. 
A
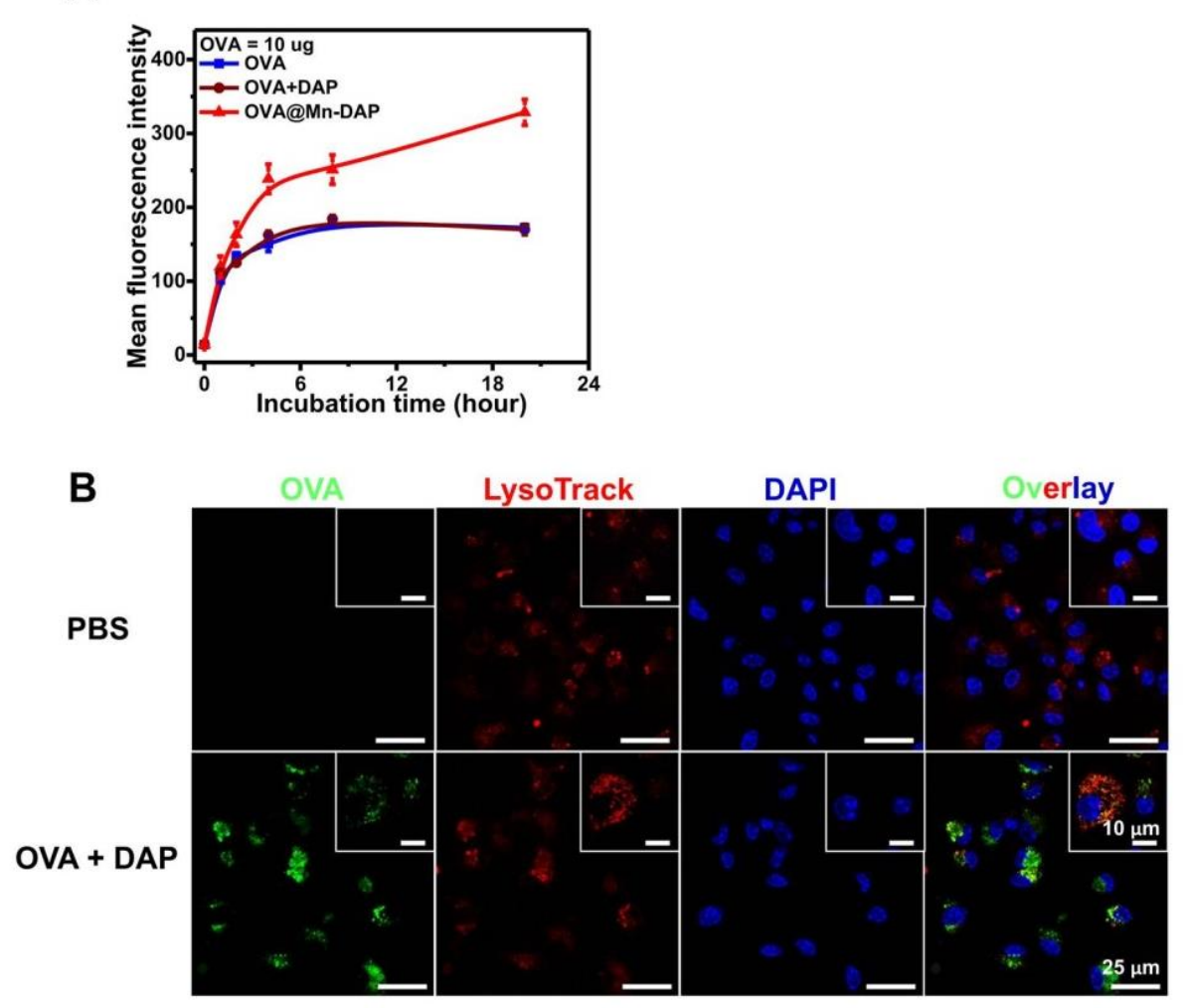

Figure S3. (A) The DC uptake of OVA in various formulations at different time points. The cellular uptake was measured by detecting fluorescence of OVA-cy5.5 using flow cytometry. (B) Confocal images of DCs after incubation with PBS or OVA-cy5.5 + DAP for $20 \mathrm{~h}$. 
A

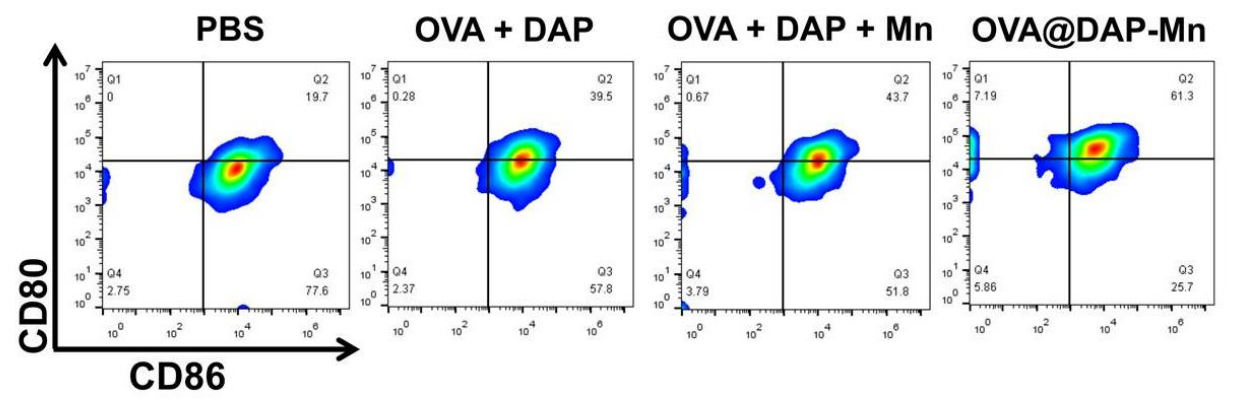

B

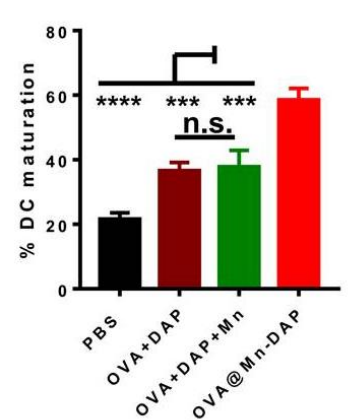

C
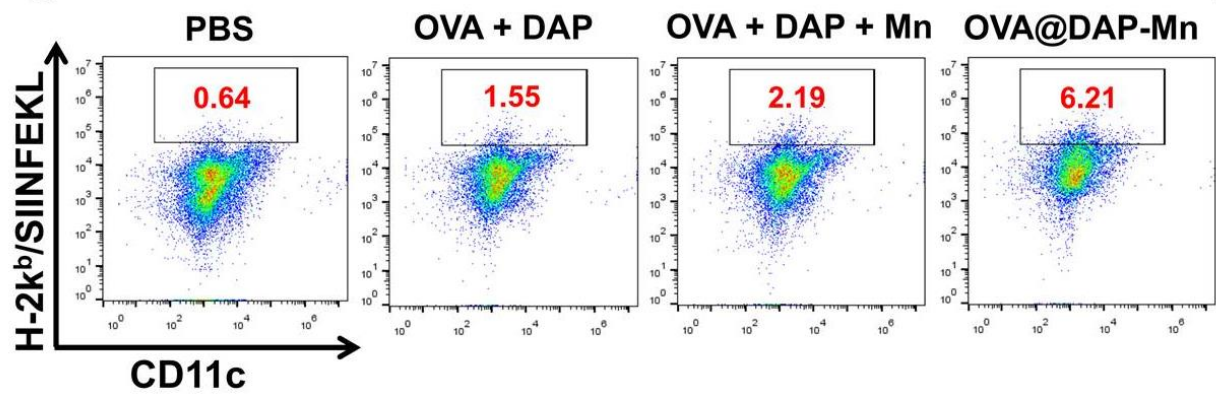

D

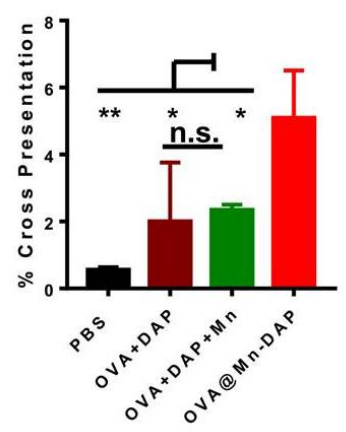

Figure S4. (A\&B) Representative flow cytometry data (A) and the statistic result (B) for DCs treated with PBS, OVA + DAP, OVA + DAP + Mn, or OVA@Mn-DAP. (C\&D) Representative flow cytometry graphs (C) and the statistic data (D) of OVA cross-presentation of DCs treated with PBS, OVA + DAP, OVA + DAP + Mn, or OVA@Mn-DAP. All the results are presented as mean \pm SD. *P $<0.05, * * \mathrm{P}<0.01, * * * \mathrm{P}<0.001, * * * * \mathrm{P}<0.0001$ and n.s. (no significant). 
A
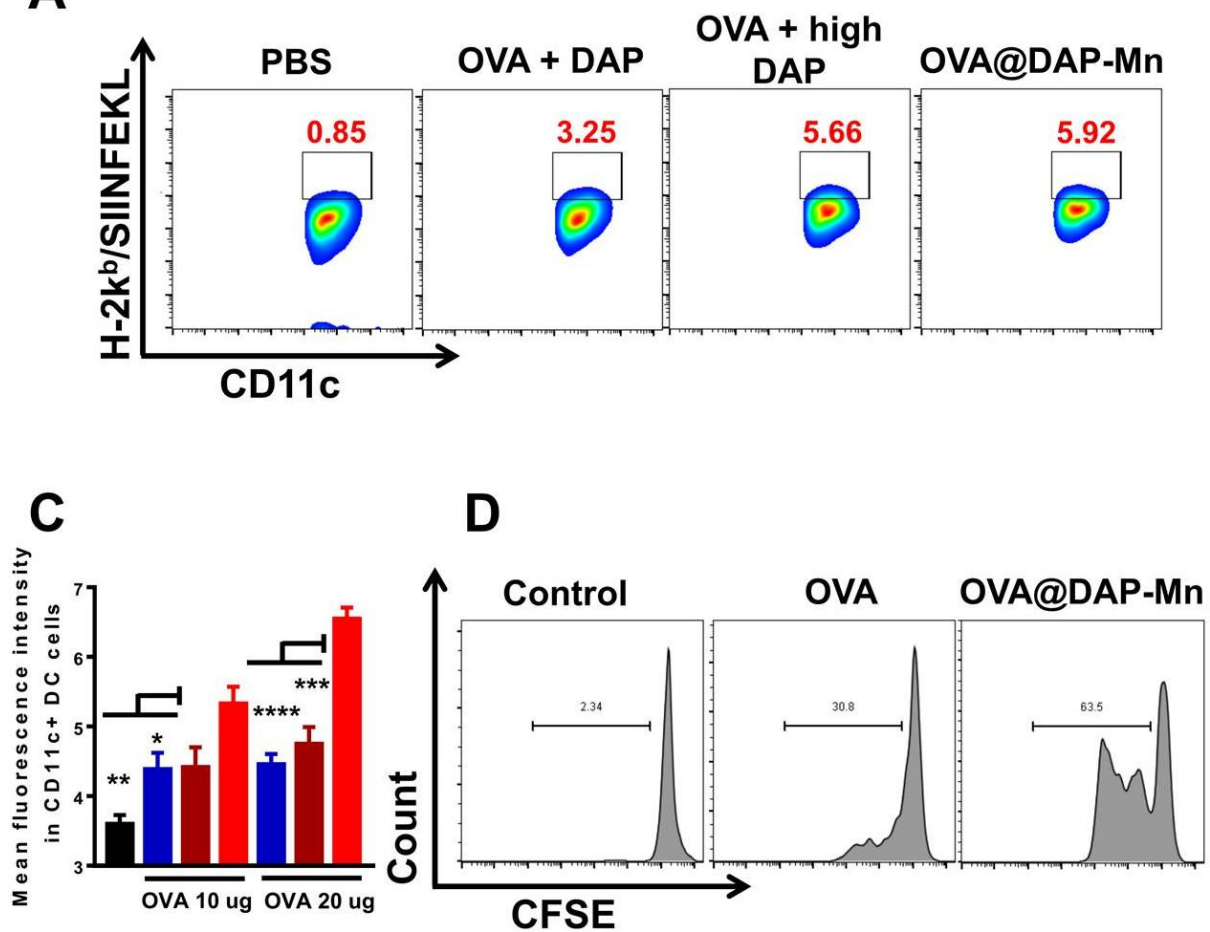

B

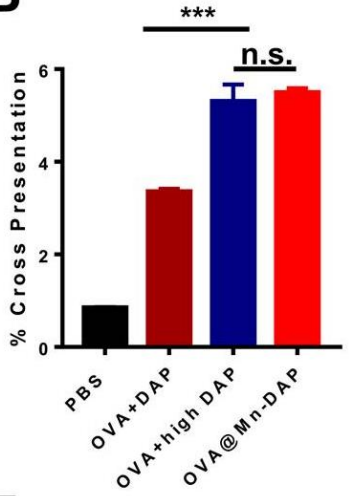

$\mathbf{E}$

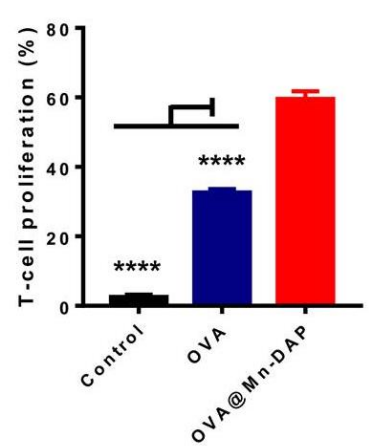

Figure S5. (A\&B) Representative flow cytometry graphs (A) and the statistic data (B) of OVA cross-presentation of DCs treated with PBS, OVA + DAP, OVA + high DAP, or OVA@Mn-DAP. The concentration of OVA and DAP was $10,11.2 \mu \mathrm{g} / \mathrm{mL}$, respectively. The mass ratio of DAP : high DAP $=1: 8 .(C)$ The mean fluorescence intensity of $\mathrm{H}-2 \mathrm{k}^{\mathrm{b}} / \mathrm{SIINFEKL}$ presentation on DCs treated with PBS, OVA, OVA + DAP or OVA@Mn-DAP for 20 h. (D\&E) Flow cytometry plots (D) and the statistical data (E) of OT-I CD ${ }^{+} \mathrm{T}$ cells proliferation after incubation with BMDCs, which were pre-stimulated with OVA or OVA@Mn-DAP (OVA $10 \mu \mathrm{g}$ ) for $20 \mathrm{~h}$ before incubation with T cells. Untreated OT-I CD $8^{+} \mathrm{T}$ cells were used as the blank control. ${ }^{* * *} \mathrm{P}<0.001,{ }^{* * * *} \mathrm{P}<0.0001$. 


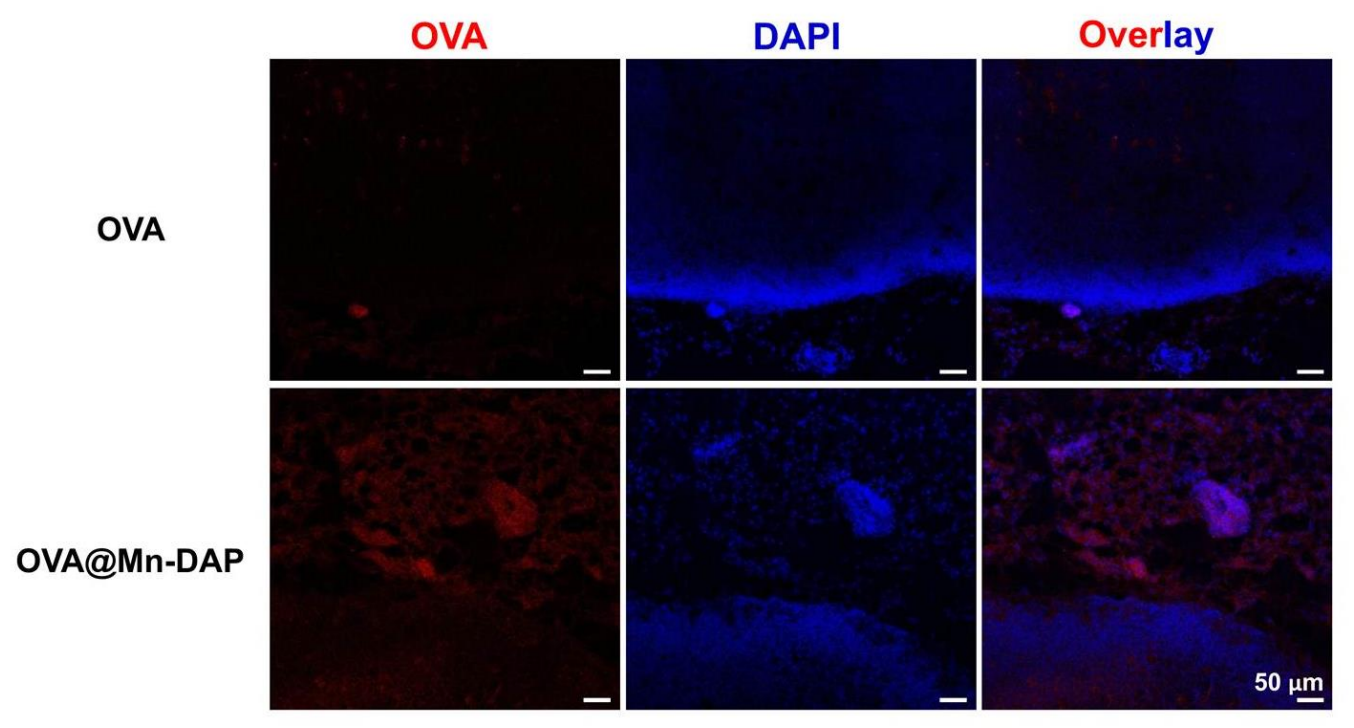

Figure S6. Confocal images of frozen sections of popliteal lymph nodes dissected from the mice injected with OVA-cy5.5 or OVA-cy5.5@Mn-DAP at 24 hours post injection. 

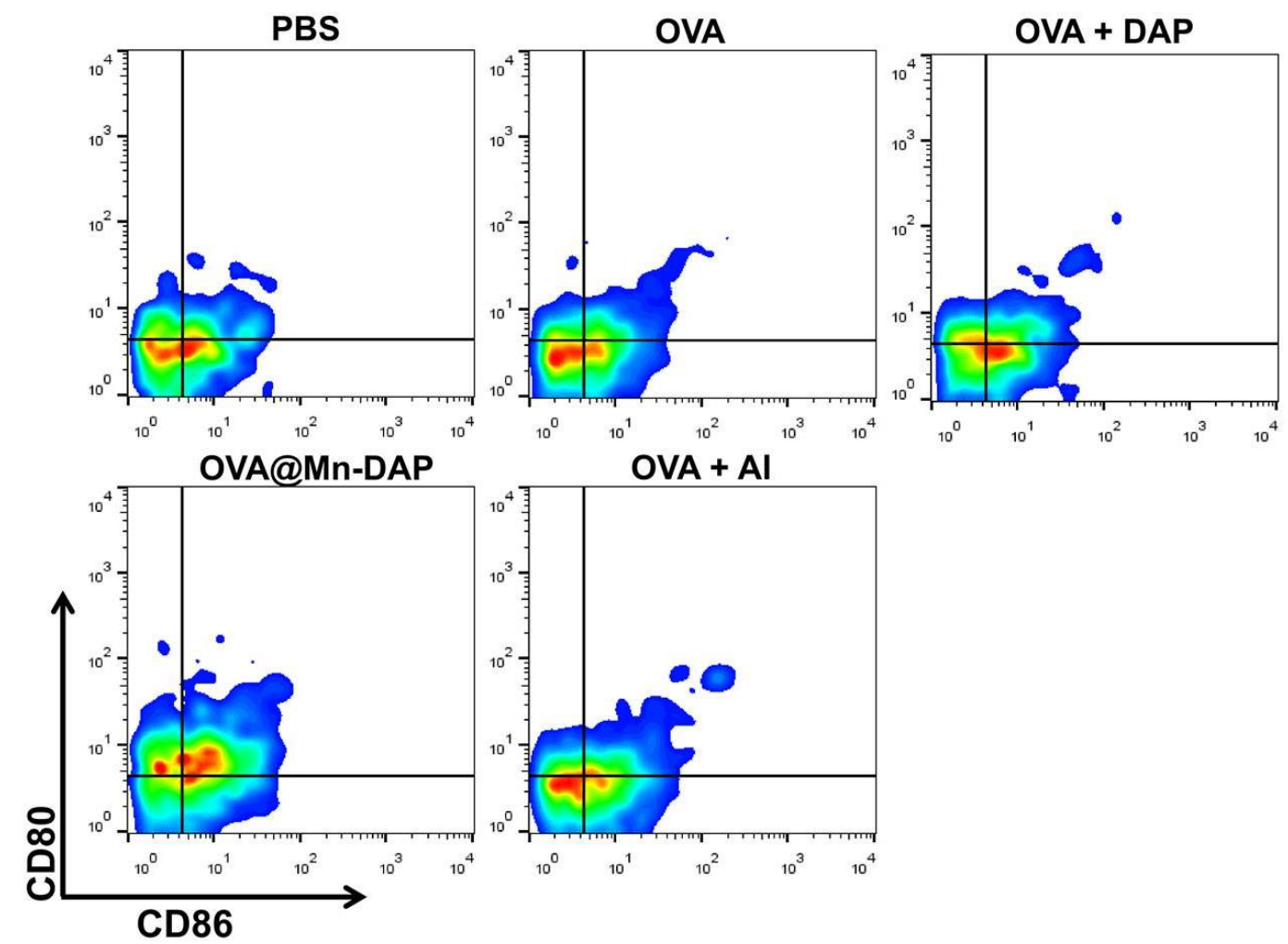

Figure S7. In vivo DC maturation promoted by different nano-vaccine formulations. Representative flow cytometry graphs for the effects of different vaccine formulations on DC maturation at day 5 post the first immunization. 

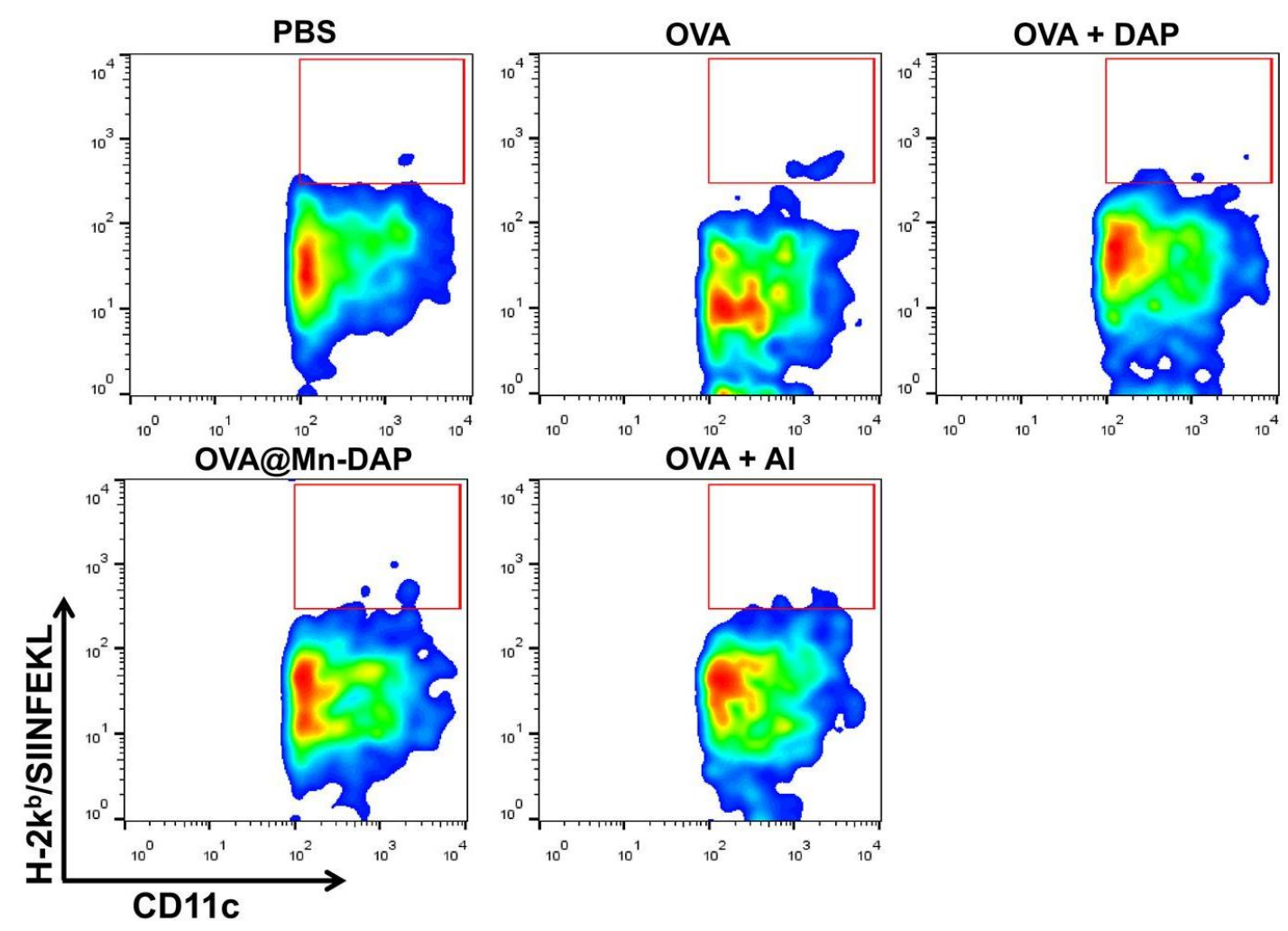

Figure S8. In vivo antigen cross-presentation induced by the vaccine formulations. Representative flow cytometry graphs of OVA antigen cross-presentation of DCs treated with various formulations. 


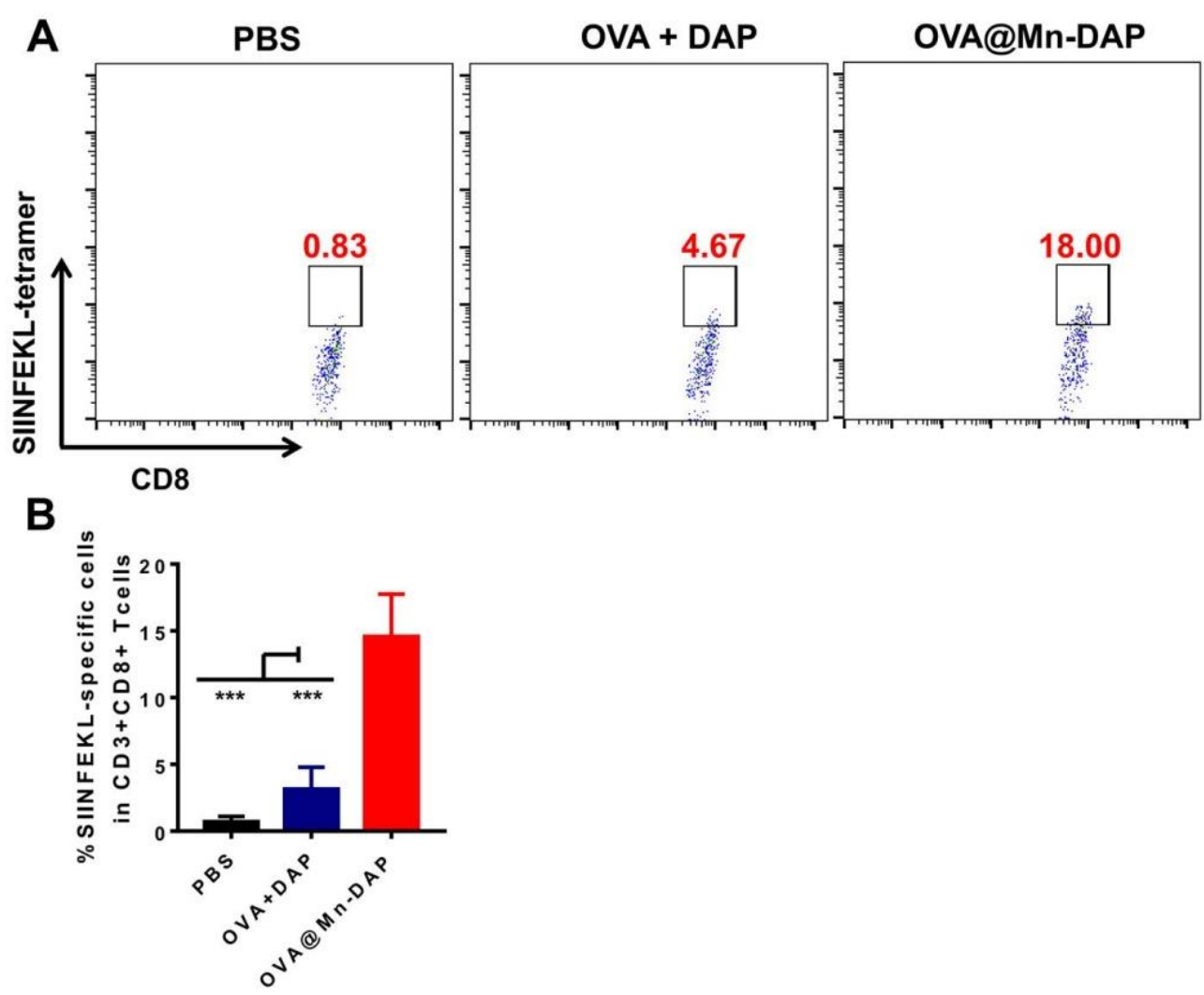

Figure S9. Flow cytometry plots (A) and the statistical data (B) of SIINFEKL-tetramer ${ }^{+} \mathrm{CD} 8^{+} \mathrm{T}$ cells from the peripheral blood of the mice vaccinated by PBS, OVA + DAP or OVA@Mn-DAP on day 21. $* * * \mathrm{P}<0.001$. 

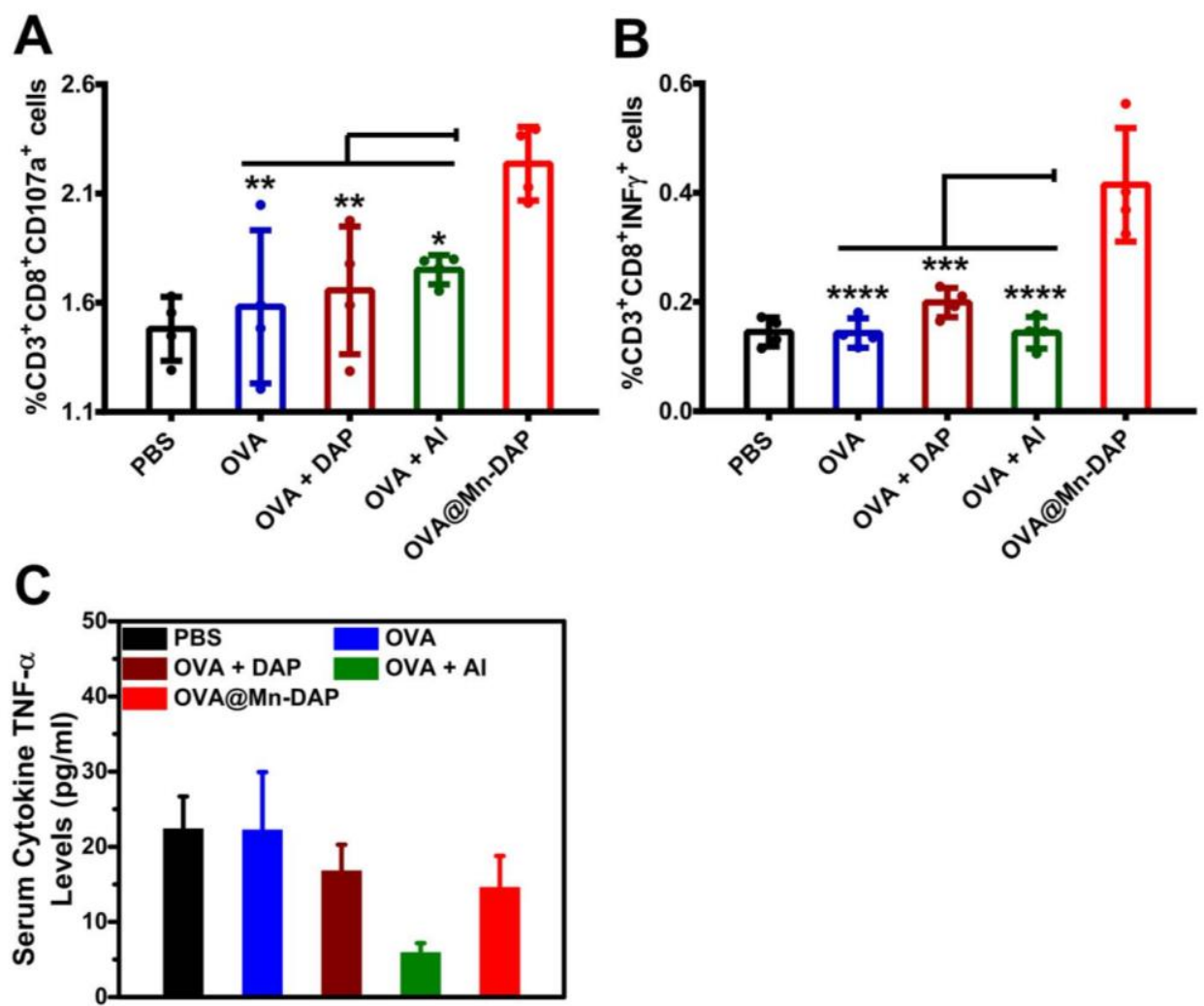

Figure S10. The percentage of $\mathrm{CD}^{+} \mathrm{CD}^{+} \mathrm{CD} 107 \mathrm{a}^{+}(\mathrm{A})$ and $\mathrm{CD} 3^{+} \mathrm{CD} 8^{+}$IFN $-\gamma^{+} \mathrm{T}$ cells (B) in the spleens after various vaccine formulations treating. (C) The levels of TNF- $\alpha$ in serum samples from mice after three doses of immunizations with various agents. Data are presented as mean $\pm \mathrm{SD} .{ }^{*} \mathrm{P}<$ $0.05, * * \mathrm{P}<0.01, * * * \mathrm{P}<0.001$ and $* * * * \mathrm{P}<0.0001$ 

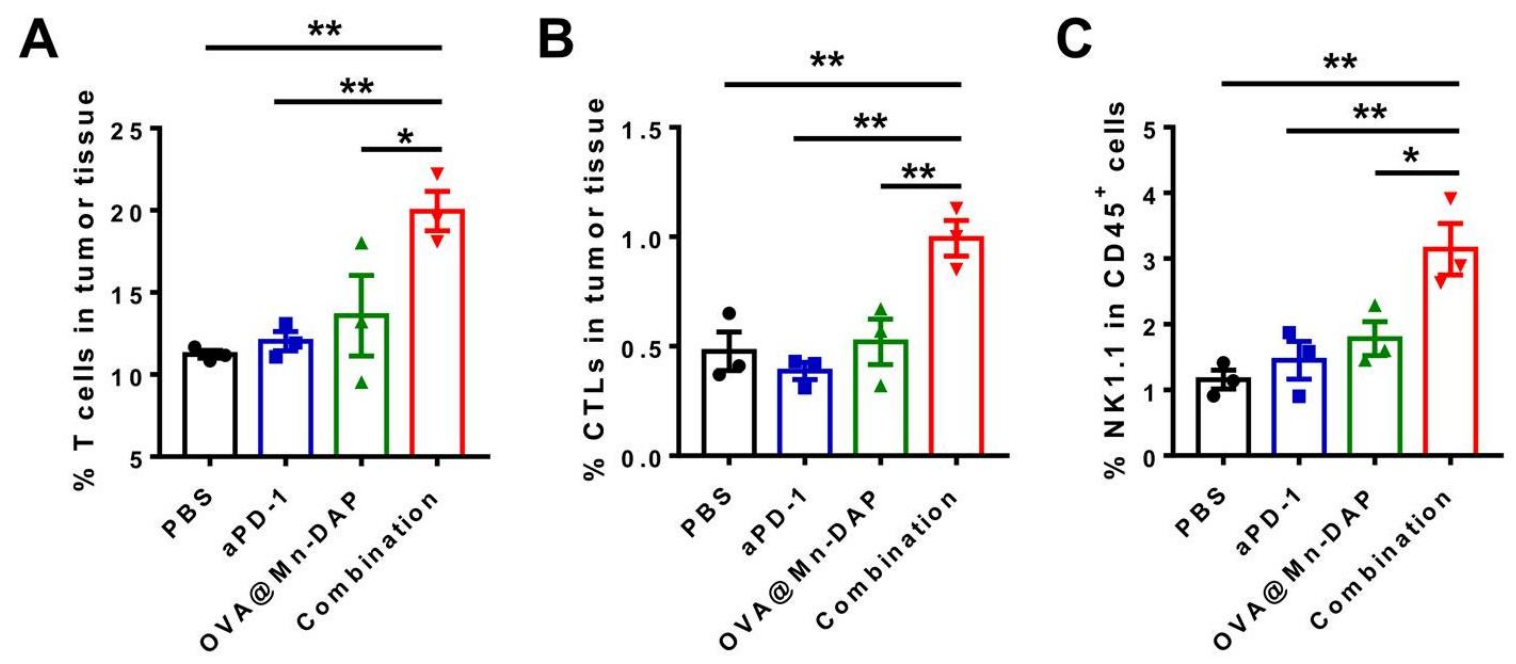

Figure S11. The percentage of T cells (A), CTLs (B) and NK cells (C) infiltration in tumors. CD3 ${ }^{+}$ cells, $\mathrm{CD}^{+} \mathrm{CD}^{+}$cells and $\mathrm{CD} 45^{+} \mathrm{NK} 1.1^{+}$cells were defined as $\mathrm{T}$ cells, CTLs and NK cells, respectively. All the results are presented as mean $\pm \mathrm{SD} . * \mathrm{P}<0.05$ and $* * \mathrm{P}<0.01$. 
A

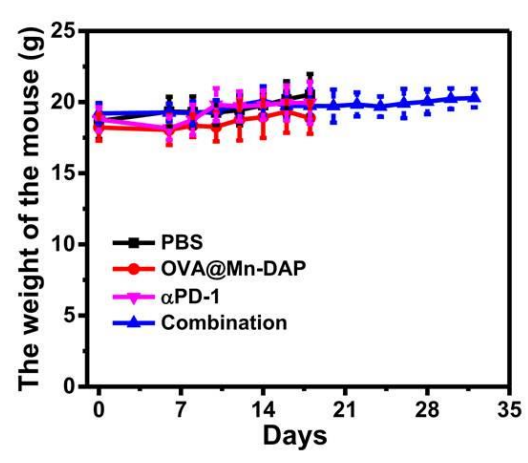

C

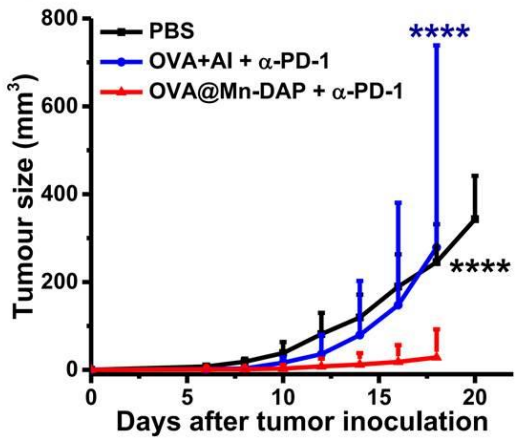

B
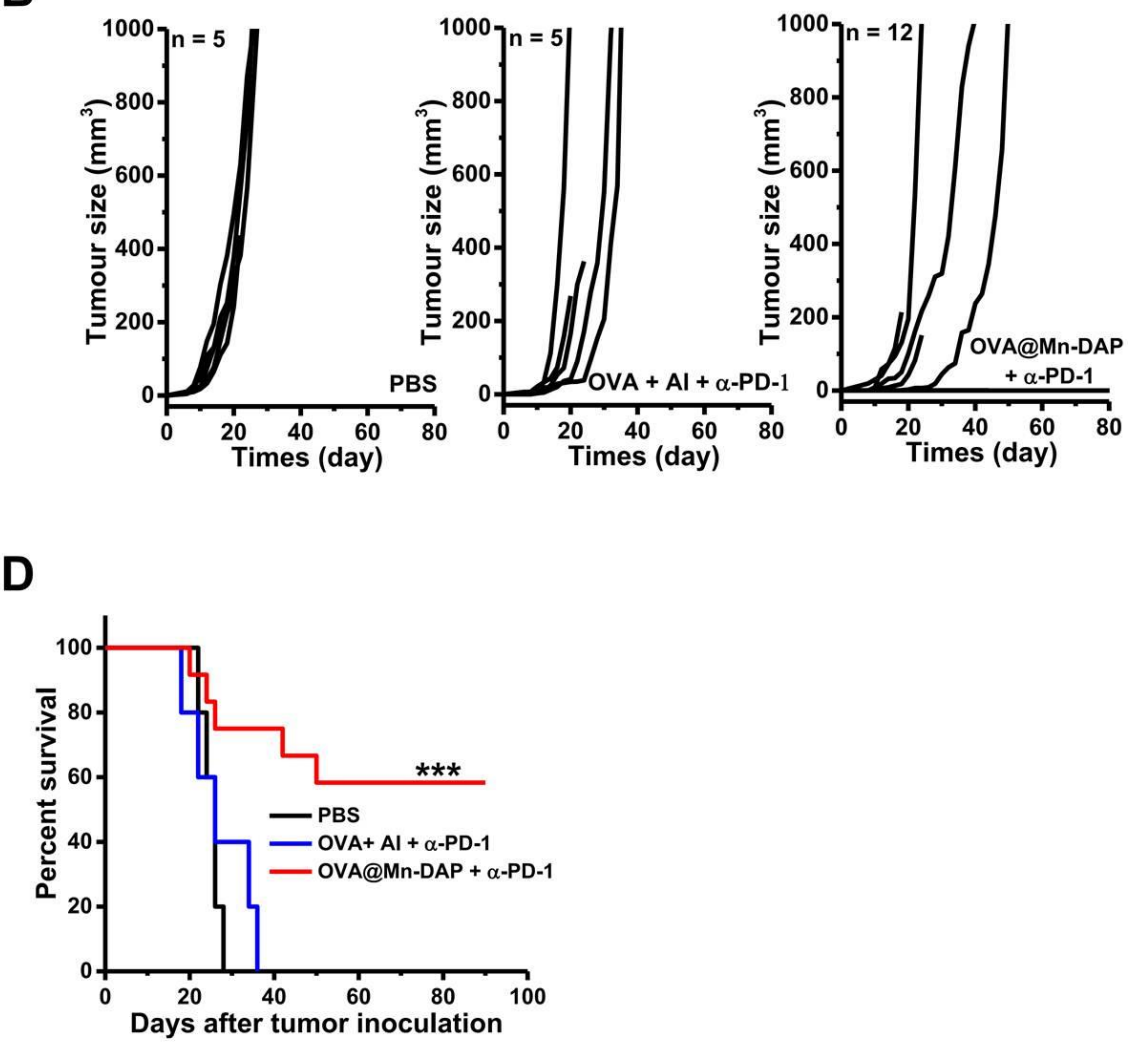

Figure S12. (A) The weight of all the mice during the vaccination. (B\&C) The individual tumor growth curves $(B)$ and average tumor growth curves $(C)$ for three groups of B16-OVA tumor-bearing mice after treated with PBS, OVA@Mn-DAP + $\alpha$-PD-1, or OVA + Al + $\alpha$-PD-1. (D) The survival data for different groups of mice as indicated. All the results are presented as mean $\pm \mathrm{SD}$. ${ }^{* * *} \mathrm{P}<$ 0.001 and $* * * * \mathrm{P}<0.0001$. 
A

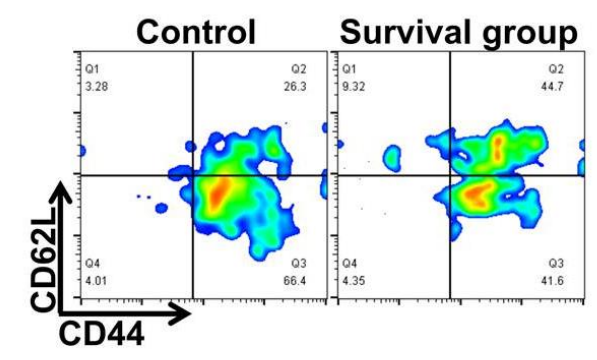

B

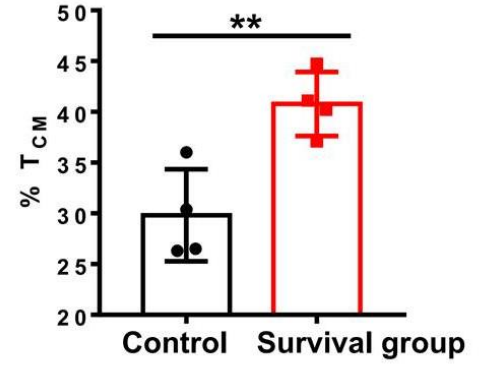

C

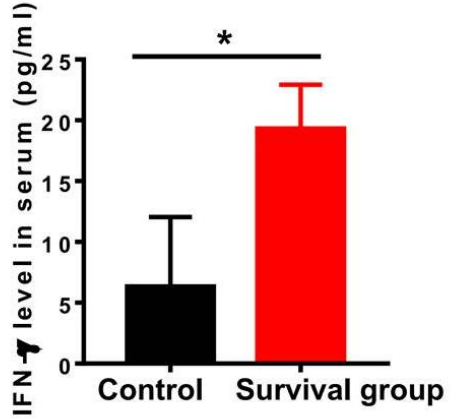

Figure S13. (A\&B) Representative flow cytometry graphs (A) and the statistic data for the percentages of $\mathrm{T}_{\mathrm{CM}}$ (B) from the two groups of mice measured on day 60 . $\mathrm{CD}^{+} \mathrm{CD} 8^{+} \mathrm{CD} 44^{+} \mathrm{CD} 62 \mathrm{~L}^{\text {high }}$ cells were defined as $\mathrm{T}_{\mathrm{CM}}$. (C) The IFN- $\gamma$ level in serum of the two groups of mice on day 60. Untreated control mice and mice survived after combination therapy to remove their first B16-OVA tumors were compared in this experiment. All the results are presented as mean $\pm \mathrm{SD}$. $* \mathrm{P}<0.05, * * \mathrm{P}<0.01$. 